\title{
The association between intravascular ultrasound-derived echo-attenuation and quantitative flow ratio in intermediate coronary lesions
}

\author{
Liang Geng ${ }^{1,2 \#}$, Yuan Yuan ${ }^{3 \#}$, Peizhao Du ${ }^{4}$, Liming Gao ${ }^{1}$, Yunkai Wang ${ }^{1}$, Jiming Li ${ }^{1}$, Wei Guo ${ }^{1}$, \\ Ying Huang ${ }^{1}$, Qi Zhang ${ }^{1}$ \\ ${ }^{1}$ Department of Cardiology, Shanghai East Hospital, School of Medicine, Tongji University, Shanghai, China; ${ }^{2}$ Department of Cardiology, JI'an \\ Hospital, Shanghai East Hospital, Ji'an, China; ${ }^{3}$ Department of Cardiology, Putuo District People's Hospital, Shanghai, China; ${ }^{4}$ Department of \\ Cardiology, Baoshan Hospital of Integrated Traditional Chinese and Western Medicine, Shanghai, China \\ Contributions: (I) Conception and design: L Geng, Q Zhang; (II) Administrative support: Q Zhang; (III) Provision of study materials or patients: \\ Y Huang, W Guo, Y Wang; (IV) Collection and assembly of data: P Du; (V) Data analysis and interpretation: L Geng, Y Yuan, L Gao, J Li; (VI) \\ Manuscript writing: All authors; (VII) Final approval of manuscript: All authors. \\ "These authors contributed equally to this work. \\ Correspondence to: Qi Zhang, MD, PhD. Department of Cardiology, Shanghai East Hospital, School of Medicine, Tongji University, Shanghai \\ 200092, China. Email: zhangqnh@hotmail.com.
}

Background: The clinical relevance of moderate coronary stenosis is determined by its morphological characteristics and physiological significance. We investigated the relationship between high-risk plaque characteristics detected by intravascular ultrasound and functional significance assessed with quantitative flow ratio (QFR) in intermediate coronary lesions.

Methods: QFR was retrospectively analyzed in 352 intermediate lesions from 330 patients undergoing intravascular ultrasound examination. The functional significance was defined as QFR $\leq 0.8$. High-risk plaque morphologies including plaque rupture, echo-lucent, echo-attenuation, and spotty calcification were identified, and attenuation indices including maximum angle, attenuation length, and superficial attenuation were determined. Clinically relevant echo-attenuation was defined as an attenuation with a minimum lumen area $\leq 4.0 \mathrm{~mm}^{2}$ and plaque burden $\geq 70 \%$.

Results: The prevalence of echo-attenuation was higher $(63.0 \%$ vs. $37.6 \%, \mathrm{P}=0.001)$ and attenuation length was longer $(12.8 \pm 10.3$ vs. $8.0 \pm 5.8 \mathrm{~mm}, \mathrm{P}=0.015)$ in lesions with $\mathrm{QFR} \leq 0.8$ compared to those with QFR $>0.8$, associated with a higher rate of clinically relevant echo-attenuation $(35.2 \% v s .10 .4 \%, \mathrm{P}<0.001)$. On multivariable analysis, the presence of echo-attenuation was an independent predictor of QFR $\leq 0.8$ [odds ratio (OR) 3.162, 95\% confidence interval (CI): 1.263-7.917, $\mathrm{P}=0.014$ ], whereas attenuation length was weakly correlated with $\mathrm{QFR}$ value $(\beta=-0.185, \mathrm{~B}=-0.002,95 \% \mathrm{CI}:-0.004$ to $-0.001, \mathrm{P}=0.001)$. Receiveroperating characteristic curve analysis revealed that the best cutoff of QFR in predicting clinically relevant echo-attenuation was 0.82 [area under the curve $(\mathrm{AUC})=0.696,95 \%$ CI: $0.616-0.775, \mathrm{P}<0.001$ ].

Conclusions: The presence of intravascular ultrasound-derived echo-attenuation confers an increased risk of QFR-defined functional significance in intermediate coronary lesions.

Keywords: Coronary artery disease; intermediate coronary lesion; quantitative flow ratio (QFR); intravascular ultrasound (IVUS); echo-attenuation

Submitted Jun 30, 2021. Accepted for publication Sep 29, 2021.

doi: $10.21037 / \mathrm{cdt}-21-402$

View this article at: https://dx.doi.org/10.21037/cdt-21-402 


\section{Introduction}

Numerous studies have demonstrated that physiologicguided percutaneous coronary intervention (PCI) is associated with better clinical outcomes, especially for intermediate lesions, which comprise a high prevalence of anatomical-functional mismatch $(1,2)$. Nevertheless, physiology is not the sole prognostic determinant, as previous intravascular ultrasound (IVUS) studies have shown that high-risk plaque morphologies, such as echoattenuation, echo-lucent and spotty calcification, were additional prognostic indicators (3-5). Therefore, a rationale combining both physiological and morphological features would be of great clinical importance in decision making and risk assessment of intermediate coronary lesions (6).

Quantitative flow ratio (QFR) has been proved to have an excellent diagnostic performance compared with fractional flow reserve (FFR) (7). Furthermore, QFR may outweigh FFR regarding the feasibility of multi-modality assessment because of several advantages, including noninvasiveness, swiftness, and adenosine-free assessment $(8,9)$. While recent studies have shown that QFR has clinical value in predicting hard cardiac events such as myocardial infarction $(10,11)$, it remains less clear as to whether its prognostic implication is related to the ability of identifying the vulnerable plaque. The current study aimed to test the hypothesis that IVUS-derived high-risk plaque morphologies are associated with QFR-defined functional severity. We presented the following article in accordance with the STARD reporting checklist (available at https://dx.doi.org/10.21037/cdt-21-402).

\section{Methods}

\section{Study design and populations}

The current study is a retrospective analysis of patients with susceptible coronary artery disease referred for coronary angiography between January 2015 and December 2018. A total of 448 patients with 482 intermediate de novo lesions [visual estimation of diameter stenosis (DS) of $50-75 \%$ and reference diameter $\geq 2.5 \mathrm{~mm}$ ] examined with IVUS were consecutively enrolled. Subjects were excluded because of poor IVUS imaging quality $(\mathrm{n}=12)$, ostial lesions $(\mathrm{n}=3)$, tandem lesions $(\mathrm{n}=12)$, collateral flow supplying from the interrogated vessel $(n=3)$, target lesions compressed by myocardial bridge $(n=5)$, left main disease $(n=6)$, vessel without 2 different projections $\left(>25^{\circ}\right)(\mathrm{n}=2)$, severe vessel overlap $(n=5)$, atrial fibrillation during angiography $(n=5)$, incomplete auto-calibrated data for QFR examination $(\mathrm{n}=77)$. Therefore, 330 patients with 352 intermediate lesions were included in the final analysis (Figure 1). Patient clinical characteristics were retrospectively acquired from a dedicated PCI database.

The study protocol was applied in accordance with the Declaration of Helsinki (as revised in 2013) and was approved by the institutional review board of Shanghai East Hospital (research project number: 2020/007). Individual consent for this retrospective analysis was waived.

\section{IVUS examination and quantitative measurements}

IVUS examination was performed after intracoronary administration of $200 \mu \mathrm{g}$ of nitroglycerin using the iLab system (Boston Scientific, Natick, Massachusetts, USA). A $40 \mathrm{MHz}$ ultrasound catheter was inserted into the interrogated vessel, then the transducer was advanced $10 \mathrm{~mm}$ distal to the target lesion and automatically pulled back at $0.5 \mathrm{~mm} / \mathrm{s}$ to the ostium. All runs were recorded for subsequent off-line analysis.

IVUS imaging analysis was made by two experienced physicians who are blinded to QFR data using a validated planimetry software (Image viewer, Boston Scientific, Natick, Massachusetts, USA). The quantitative IVUS measurements were performed according to the American College of Cardiology Clinical Expert Consensus Document on Standards for Acquisition, Measurement and Reporting of IVUS Studies (12). Briefly, external elastic membrane (EEM) cross-sectional area (CSA), lumen CSA, and plaque plus media $(\mathrm{P}+\mathrm{M})$ CSA were automatically traced at the lesion site and $5 \mathrm{~mm}$ proximal and distal reference sites. The lesion site was defined as the site with the minimum lumen area (MLA). The reference site was defined as the site with the largest lumen and smallest plaque burden $(\mathrm{PB})$ without any intervening side branch. Area stenosis (AS, \%) was calculated as (mean reference lumen CSA - MLA)/mean reference lumen CSA. PB (\%) was derived from following formula: $\mathrm{PB}=(\mathrm{P}+\mathrm{M}) \mathrm{CSA} /$ EEM CSA. Remodeling index (RI) was calculated as the EEM CSA at the lesion site divided by the average of the proximal and distal reference segment EEM CSAs. Plaque eccentricity was calculated as (maximum - minimum plaque thickness)/maximum plaque thickness.

\section{IVUS-derived high-risk plaque morphologies}

The following IVUS-derived high-risk plaque morphologies which have been associated with plaque vulnerability and 


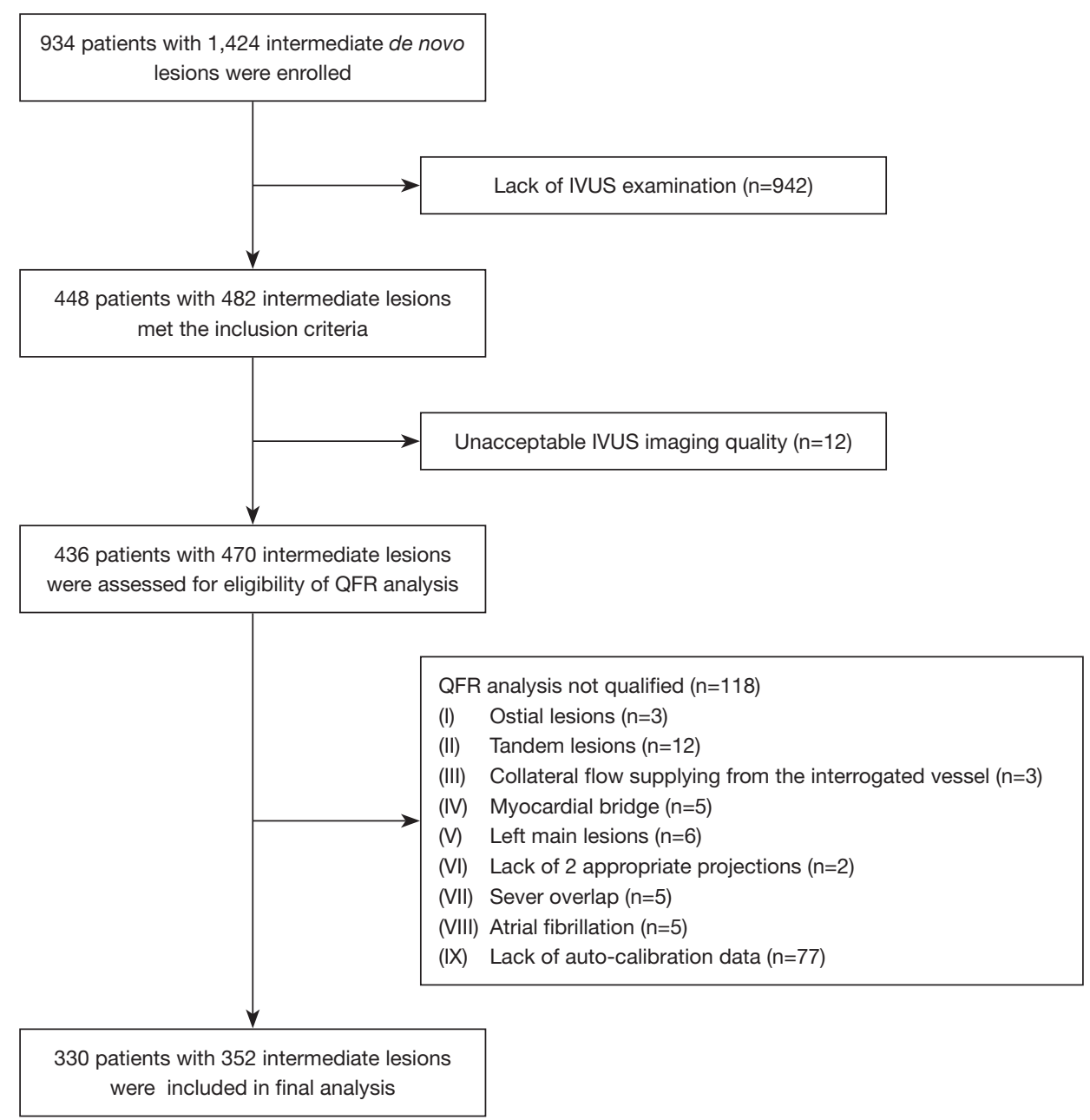

Figure 1 Flowchart of patient enrollment. N, lesion number; QFR, quantitative flow ratio; IVUS, intravascular ultrasound.

worse clinical outcomes were assessed (4,5,13): plaque rupture was defined as the presence of a cavity that communicated with the lumen. Positive remodeling was defined as a RI of $>1.05$. Echo-attenuation was defined as the loss of ultrasound signal behind plaque in the absence of calcification (3). The maximum attenuation angle was measured in degrees with a protractor centered on the lumen. The attenuation length was measured on the longitudinal view. The location of echoattenuation was defined as superficial or deep as previously described (3). The echo-attenuation with an MLA $\leq 4 \mathrm{~mm}^{2}$ and with a $\mathrm{PB} \geq 70 \%$ was considered clinically relevant (14). Echo-lucent was defined as the absence of ultrasound signal within the plaque (3). Spotty calcification was defined as the plaque with calcium deposits within arcs of $<90^{\circ}$ (3). Discordance between the two investigators regarding the qualitative assessment was resolved by a consensus reading.
The inter- and intra- observer coefficient $\kappa$ indices for echo-attenuation, echo-lucent and spotty calcification were $0.932,0.772$, and 0.880 , and $0.966,0.870$ and 0.920 , respectively.

\section{QFR assessment}

QFR was retrospectively analyzed by two qualified investigators who were blinded to the IVUS data using the validated QFR system (AngioPlus, Pulse Medical Imaging Technology, Shanghai Co., Ltd., Shanghai, China). The QFR calculation process was performed as previously validated algorithm (7). Briefly, three-dimensional model was reconstructed by automatically delineating the lumen contour in two appropriate projections with different angles $\left(\geq 25^{\circ}\right)$ of interrogated vessel. Manual correction was limited 
and only permitted in case of side-branch or sub-optimal image quality. Contrast flow model using the frame count method was introduced to derive the final QFR value. QFR $\leq 0.8$ was considered as functionally significant. Threedimensional quantitative coronary angiography (3D-QCA) parameters, including reference vessel diameter (RVD), DS (\%), minimal lumen diameter (MLD), and lesion length were automatically derived from the final QFR report.

\section{Statistical analysis}

The statistical analyses were performed with $\mathrm{R}$ software, version 4.0.4. ( $\mathrm{R}$ foundation for Statistical Computing, Vienna, Austria), SPSS version 22.0 (SPSS institute, Chicago, Illinois, USA) and GraphPad prism 7.00 (GraphPad Software, Inc., La Jolla, California, USA). Continuous variables are expressed as mean \pm standard deviation and were compared using Student's $t$-test. Categorical variables are expressed as frequencies (percentages) and were compared using chi-square test. Intra-observer and inter-observer differences were evaluated using Cohen's $\kappa$ coefficient. The correlations between continuous parameters were assessed using Pearson correlation analysis. The independent correlation between continuous QFR value and IVUS parameters were assessed using multivariable liner regression analysis. Independent predictors of QFR $\leq 0.8$ were evaluated using multivariable logistic regression which was performed after forward selection of clinical risk factors, QCA and IVUS parameters, with significance for addition to the model set at $\mathrm{P} \leq 0.15$. Variables with significance levels of $\mathrm{P}<0.1$ in univariable analysis were considered for multivariable analysis. Receiver operating characteristic (ROC) curves were introduced to identify the optimal cutoff value of IVUS parameters to predict the functional significance and to determine the best cutoff of QFR to predict the presence of echo-attenuation with clinical relevance. The comparisons of area under the curve (AUC) were performed using the Delong method (15). A two-sided $\mathrm{P}$ value $<0.05$ was considered statistically significant.

\section{Results}

\section{Baseline characteristics}

Overall, 276 patients had QFR $>0.8$ and 54 had QFR $\leq 0.8$. The two groups did not differ with respect to demographics, clinical characteristics, and risk factors for coronary artery disease, except for a higher incidence of multi-vessel disease and higher triglyceride levels in patients with QFR $\leq 0.8$ (Table 1).

\section{Lesion characteristics}

Compared to lesions with QFR $>0.8 \quad(\mathrm{n}=298)$, those with QFR $\leq 0.8 \quad(n=54)$ were more frequently located in left anterior descending artery, had higher DS, longer lesion length and smaller MLD and MLA. Furthermore, AS, PB and eccentricity were higher in lesions with $\mathrm{QFR} \leq 0.8$, but RVD and RI were similar between the two groups (Table 2).

The prevalence of echo-attenuation $(63.0 \%$ vs. $37.6 \%$, $\mathrm{P}=0.001)$ and clinically relevant echo-attenuation $(35.2 \%$ vs. $10.4 \%, \mathrm{P}<0.001$ ) were higher in lesions with $\mathrm{QFR} \leq 0.8$ versus those with $\mathrm{QFR}>0.8$. When the attenuation indices were compared according to QFR, only attenuation length was significantly different between the two lesion groups $(12.8 \pm 10.3$ vs. $8.0 \pm 5.8 \mathrm{~mm}, \mathrm{P}=0.015)$. On the contrary, there were no significant differences between the two groups in plaque rupture, positive remodeling, echo-lucent and spotty calcification (Table 2).

\section{Association between echo-attenuation and QFR}

A representative illustration of echo-attenuation with QFR $\leq 0.8$ is demonstrated in Figure 2. There was a significant albeit weak correlation between the maximum attenuation angle and QFR ( $\mathrm{r}=-0.181, \mathrm{P}=0.029)$ (Figure 3A), and a moderate correlation between attenuation length and QFR $(\mathrm{r}=-0.316, \mathrm{P}<0.001)$ (Figure $3 \mathrm{~B})$. The comparisons of AUCs between attenuation indices and anatomical references (MLA and AS) for predicting QFR $\leq 0.8$ are shown in Figure 4. The differences between attenuation length $(\mathrm{AUC}=0.639)$ and anatomical references $(\mathrm{AUC}=0.698$ for MLA, $\mathrm{P}=0.429$; $\mathrm{AUC}=0.634$ for $\mathrm{AS}, \mathrm{P}=0.946$ ) were not statistically significant. Similar results were observed between attenuation angle ( $\mathrm{AUC}=0.591$ ) and anatomical references $(\mathrm{P}=0.129$ for MLA; $\mathrm{P}=0.536$ for $\mathrm{AS})$.

When adjusting for IVUS parameters and other anatomical confounders, only attenuation length was correlated independently with QFR $[\beta=-0.185, \mathrm{~B}=-0.002$, $95 \%$ confidence interval $(\mathrm{CI}):-0.004$ to $-0.001, \mathrm{P}=0.001]$ (Table 3). Echo-attenuation [odds ratio (OR) 3.162, 95\% CI: 1.263-7.917, $\mathrm{P}=0.014]$, MLA (OR 1.749, 95\% CI: 1.099 2.784, $\mathrm{P}=0.018)$ and DS (OR 1.357, 95\% CI: 1.236-1.489, $\mathrm{P}<0.001)$, were identified as the independent predictors of QFR-defined functional significance (Table 4). ROC analysis showed that the best cutoff of QFR in predicting clinically 
Table 1 Clinical characteristics as stratified by QFR $=0.8$

\begin{tabular}{|c|c|c|c|}
\hline Characteristics & QFR $>0.8(n=276)$ & QFR $\leq 0.8(n=54)$ & $P$ value \\
\hline Male, n (\%) & $160(58.0)$ & $33(61.1)$ & 0.663 \\
\hline Hypertension, n (\%) & $184(66.7)$ & $38(70.4)$ & 0.596 \\
\hline Hypercholesterolemia, n (\%) & $11(4.0)$ & $5(9.3)$ & 0.099 \\
\hline Current Smoker, n (\%) & $100(36.2)$ & $16(29.6)$ & 0.353 \\
\hline Acute coronary syndrome, $\mathrm{n}(\%)$ & $11(4.0)$ & $4(7.4)$ & 0.270 \\
\hline Multi-vessel disease, n (\%) & $153(55.4)$ & $38(70.4)$ & 0.045 \\
\hline Prior PCI history, n (\%) & $37(13.4)$ & $10(18.5)$ & 0.326 \\
\hline LVEF (\%) & $62.7 \pm 4.2$ & $62.7 \pm 3.5$ & 0.950 \\
\hline $\mathrm{BMI}, \mathrm{kg} / \mathrm{m}^{2}$ & $24.2 \pm 3.9$ & $24.0 \pm 2.7$ & 0.830 \\
\hline \multicolumn{4}{|l|}{ Laboratory } \\
\hline Creatinine, $\mu \mathrm{mol} / \mathrm{L}$ & $72.9 \pm 17.8$ & $73.9 \pm 20.0$ & 0.720 \\
\hline LDL-C, $\mathrm{mmol} / \mathrm{L}$ & $2.5 \pm 0.9$ & $2.6 \pm 1.0$ & 0.543 \\
\hline $\mathrm{TG}, \mathrm{mmol} / \mathrm{L}$ & $1.4 \pm 0.8$ & $1.7 \pm 1.0$ & 0.045 \\
\hline HBA1c, \% & $6.2 \pm 1.3$ & $6.5 \pm 1.4$ & 0.299 \\
\hline Prior statin treatment, $\mathrm{n}(\%)$ & $42(15.2)$ & $11(20.4)$ & 0.417 \\
\hline
\end{tabular}

Values are mean \pm standard deviation or number (percentage). QFR, quantitative flow ratio; $\mathrm{PCl}$, percutaneous coronary intervention; CABG, coronary artery bypass graft; MI, myocardial infarction; LVEF, left ventricular eject fraction; BMI, body mass index; LDL-C, lowdensity lipoprotein cholesterol; TG, triglyceride; HBA1c, hemoglobin A1c.

relevant echo-attenuation was 0.82 (AUC $=0.696,95 \%$ CI: $0.616-0.775, \mathrm{P}<0.001$ ) (Figure 5).

\section{Discussion}

The results of this study showed that in intermediate coronary lesions, presence of echo-attenuation was an independent morphological determinant of QFR-defined functional significance. QFR $\leq 0.82$ was useful in predicting clinically relevant echo-attenuation.

\section{Plaque morphologies and physiological significance}

Classic anatomical parameters, from plain angiographyderived DS to IVUS-derived MLA and PB, have long been considered as the main determinants of functional significance of coronary stenotic lesions $(16,17)$. In contrast, the relationships between plaque morphology and functional significance were less investigated and varied greatly among different imaging or relevant hemodynamic modalities. Previous virtual histology (VH)-IVUS studies failed to establish the association between plaque morphology and functional significance $(18,19)$, mainly because of limited reproducibility of this technique (20). On the other hand, coronary computed tomography angiography (CCTA)derived adverse plaque characteristics were independently correlated with FFR but not with positron emission tomography-derived myocardial blood flow or instantaneous wave-free ratio $(21,22)$.

The major finding of the current study was that there existed a significant correlation between echoattenuation and QFR-defined functional significance. It has been demonstrated that $91.4 \%$ of echo-attenuation detected by IVUS and near-infrared spectroscopy (NIRS) 
Table 2 Lesion characteristics as stratified by QFR $=0.8$

\begin{tabular}{|c|c|c|c|}
\hline Characteristics & QFR $>0.8(n=298)$ & QFR $\leq 0.8(n=54)$ & $P$ value \\
\hline Non-culprit lesions, n (\%) & $12(4.0)$ & $4(7.4)$ & 0.272 \\
\hline \multicolumn{4}{|l|}{ 3D-QCA parameters, n (\%) } \\
\hline LAD lesions & $207(69.5)$ & $50(92.6)$ & $<0.001$ \\
\hline $\mathrm{RVD}, \mathrm{mm}$ & $3.2 \pm 0.6$ & $3.3 \pm 0.6$ & 0.422 \\
\hline $\mathrm{DS}, \%$ & $38.9 \pm 7.5$ & $51.0 \pm 5.7$ & $<0.001$ \\
\hline Lesion length, mm & $23.3 \pm 12.2$ & $31.7 \pm 13.6$ & $<0.001$ \\
\hline MLD, mm & $1.9 \pm 0.5$ & $1.6 \pm 0.3$ & $<0.001$ \\
\hline AS, \% & $57.8 \pm 11.5$ & $64.8 \pm 10.3$ & $<0.001$ \\
\hline PB, \% & $65.3 \pm 8.8$ & $70.0 \pm 9.1$ & $<0.001$ \\
\hline Eccentricity & $0.76 \pm 0.17$ & $0.81 \pm 0.15$ & 0.026 \\
\hline $\mathrm{RI}$ & $0.86 \pm 0.20$ & $0.83 \pm 0.20$ & 0.250 \\
\hline \multicolumn{4}{|l|}{ High risk IVUS features, n (\%) } \\
\hline Plaque rupture & $20(6.7)$ & $2(3.7)$ & 0.398 \\
\hline Positive remodeling & $51(17.1)$ & $6(11.1)$ & 0.271 \\
\hline Echo-lucent & $108(36.2)$ & $20(37.0)$ & 0.775 \\
\hline Attenuation length, $\mathrm{mm}$ & $8.0 \pm 5.8$ & $12.8 \pm 10.3$ & 0.015 \\
\hline Superficial attenuation, $\mathrm{n}(\%)$ & $86(76.8)$ & $27(79.4)$ & 0.748 \\
\hline
\end{tabular}

Values are mean \pm standard deviation or number (percentage). ${ }^{\dagger}$, echo-attenuation with an MLA $\leq 4.0 \mathrm{~mm}^{2}$ and with a PB $\geq 70 \%$. QFR, quantitative flow ratio; $\mathrm{PCl}$, percutaneous coronary intervention; 3D-QCA, 3-dimensional quantitative coronary angiography; LAD, left anterior descending; RVD, reference vessel diameter; DS, diameter stenosis; MLD, Minimum lumen diameter; IVUS, intravascular ultrasound; MLA, minimum lumen area; AS, area stenosis; PB, plaque burden; RI, remodeling index.

are histo-pathologically related to lipid-rich necrotic core or lipid pool $(3,23)$. Hence, the inherent lipid-rich nature underscores the vulnerable characteristic of echoattenuation. Consistent with our finding, recent optical coherence tomography (OCT) studies showed that OCTderived thin-cap fibroatheroma (TCFA) was correlated with lower FFR and QFR (24,25). In contrast, previous in-vitro computational flow dynamics study showed that lipid-rich plaque was related to higher FFR value, owing to the greater deformation of the lumen under the stress (26). Therefore, computational FFR value may be underestimated in the lipid-rich lesion as the rigid vessel walls are universally assumed in all lesions during the angiography-based geometry reconstruction (27). On the other hand, recent 


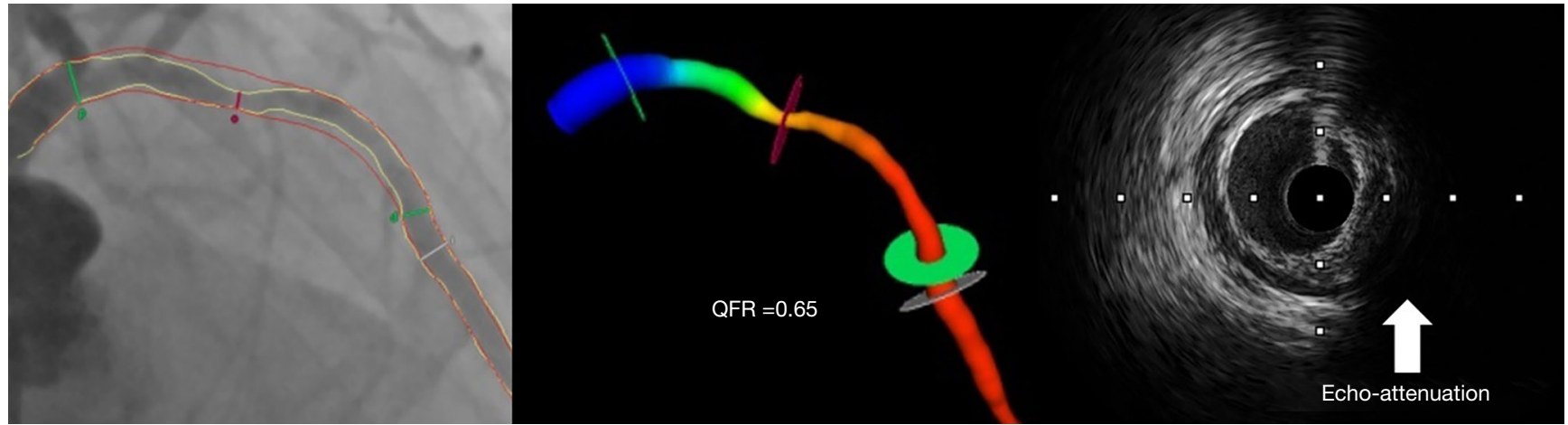

Figure 2 Representative illustration of an echo-attenuation with QFR-defined functional significance. QFR, quantitative flow ratio.

A

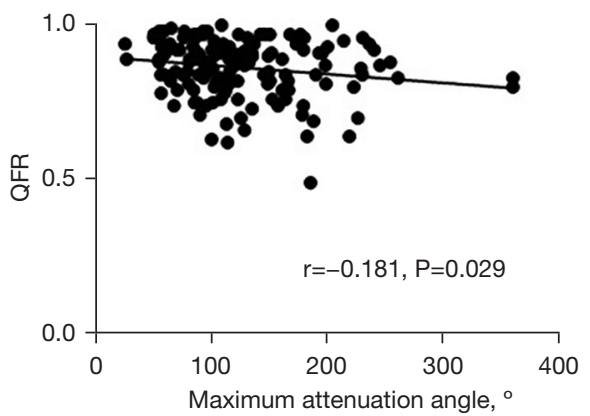

B

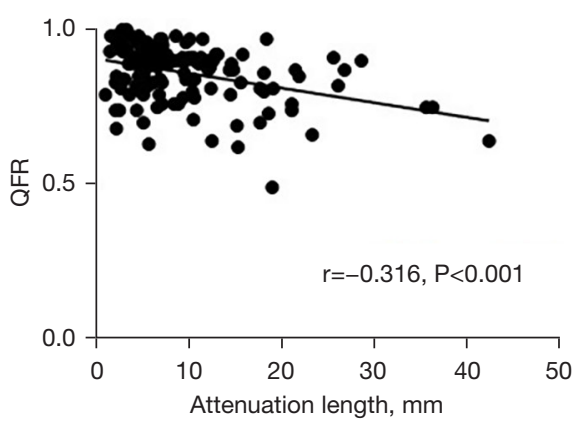

Figure 3 Negative correlations between attenuation indices and QFR. (A) Maximum attenuation angle; (B) attenuation length. QFR, quantitative flow ratio.

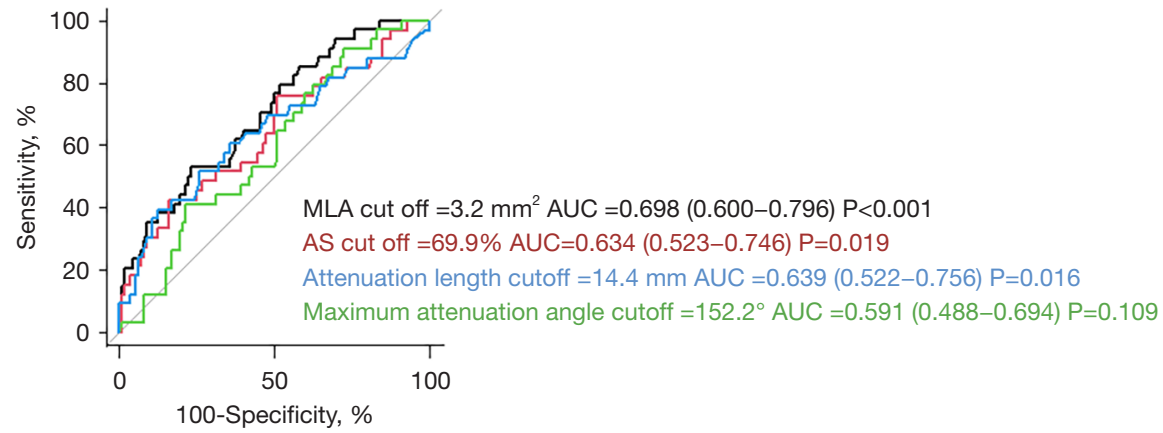

Figure 4 The ROC curve analysis of IVUS parameters for predicting QFR $\leq 0.8$. AS, area stenosis; AUC, area under the curve; MLA, minimum lumen area; ROC, receiver operating characteristic; IVUS, intravascular ultrasound; QFR, quantitative flow ratio.

NIRS study has shown that lipidic plaques may negatively affect the FFR value through hampering the hyperemiainduced vasoreactivity (28). Further studies incorporating computational flow dynamics analysis, resistance/flow measurements and intravascular imaging are warranted to further explore the mechanism explaining the physiologic relevance of lipidic plaque component.

In this study, maximum attenuation angle and attenuation length were correlated weakly with QFR, and the diagnostic performance of attenuation indices in predicting the functional significance was not superior to that of classical IVUS-derived anatomical parameters. Furthermore, only 
Table 3 Association between QFR and attenuation indices in multiple liner regression analysis

\begin{tabular}{|c|c|c|c|c|}
\hline Variables & $\beta$ & B & $95 \% \mathrm{Cl}$ & Pvalue \\
\hline AS, $\%$ & -0.082 & -0.065 & -0.167 to 0.036 & 0.203 \\
\hline PB, \% & -0.045 & -0.001 & -0.002 to 0.001 & 0.451 \\
\hline DS, \% & -0.555 & -0.006 & -0.007 to -0.005 & $<0.001$ \\
\hline Lesion length & -0.187 & -0.001 & -0.002 to -0.001 & $<0.001$ \\
\hline LAD lesions & -0.282 & -0.061 & -0.083 to -0.038 & $<0.001$ \\
\hline Multi-vessel disease & -0.064 & -0.012 & -0.033 to 0.008 & 0.228 \\
\hline Superficial attenuation & 0.033 & 0.007 & -0.015 to 0.029 & 0.513 \\
\hline
\end{tabular}

QFR, quantitative flow ratio; Cl, confidence interval; MLA, minimum lumen area; AS, area stenosis; PB, plaque burden; DS, diameter stenosis; LAD, left anterior descending.

Table 4 Multivariable logistic regression for the prediction of $\mathrm{QFR} \leq 0.8$

\begin{tabular}{lcc}
\hline Variables & Odds ratio $(95 \% \mathrm{Cl})$ & Pvalue \\
\hline Multi-vessel disease & $0.469(0.178-1.235)$ & 0.126 \\
LAD lesions & $3.228(0.837-12.450)$ & 0.089 \\
Eccentricity, per 0.1 increase & $1.276(0.992-1.641)$ & 0.058 \\
MLA, per $0.1 \mathrm{~mm}^{2}$ decrease & $1.749(1.099-2.784)$ & 0.018 \\
Lesion length, per 1 mm increase & $1.027(0.993-1.062)$ & 0.121 \\
Diameter stenosis, per 1\% increase & $1.357(1.236-1.489)$ & $<0.001$ \\
Echo-attenuation & $3.162(1.263-7.917)$ & 0.014 \\
\hline
\end{tabular}

QFR, quantitative flow ratio; $\mathrm{Cl}$, confidence interval; LAD, left anterior descending; MLA, minimum lumen area.

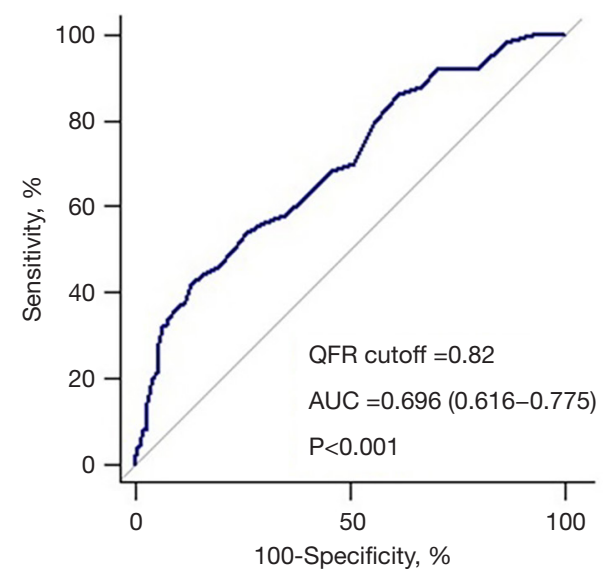

\begin{tabular}{|l|c|}
\hline Accuracy, \% & 80.7 \\
\hline Sensitivity, \% & 42.0 \\
\hline Specificity, \% & 87.1 \\
\hline Positive predictive value, \% & 35.0 \\
\hline Negative predictive value, \% & 90.1 \\
\hline
\end{tabular}

Figure 5 The ROC Curve analysis of QFR for predicting clinically relevant echo-attenuation. AUC, area under the curve; QFR, quantitative flow ratio; ROC, receiver operating characteristic. 
attenuation length was independently correlated with QFR in the multivariable liner regression analysis, suggesting that the size of echo-attenuation may be not the main contributor of functional significance. Further studies are needed to prove this issue.

In the current study, no other high-risk plaque morphologies were found to be correlated with QFR $\leq 0.8$ except for echo-attenuation. In contrast, previous CCTA studies have demonstrated that positive remodeling and spotty calcification were associated with abnormal FFR $(21,22)$. The explanations of this discrepancy are more likely multifactorial. First, different imaging modalities were used in previous studies. Second, positive remodeling, as a compensatory response to prevent lumen loss caused by plaque, may exert a positive impact on QFR value. Third, spotty calcification is correlated with a lower grade of lumen stenosis (29), which could decrease its effect on functional severity. Finally, compared with echo-attenuation, both spotty calcification and echo-lucent have limited accuracy in identifying lipid-rich plaque and, thus, may also have limitations in predicting functionally significant lesions (3).

\section{Potential clinical implication}

Initially, echo-attenuation was considered as a predictor of periprocedural myocardial injury and no-reflow phenomenon during PCI (30,31). A longitudinal IVUS study demonstrated that presence and progression of echo-attenuation were predictors of long-term cardiovascular events (5). The present study provides an additional insight into the clinical importance of echo-attenuation by linking physiological stenosis severity to this high-risk IVUS signature.

Notably, our study showed that despite the significant p-value, QFR $\leq 0.82$ predicted clinically relevant echoattenuation in intermediate coronary lesions, with a moderate AUC of 0.696. Likewise, although the negative predictive value of $\mathrm{QFR}>0.82$ to preclude the clinically relevant echo-attenuation was high (90.1\%), IVUS and QFR may complement each other in certain clinical scenarios with relatively higher prevalence of vulnerable plaque, such as diabetes mellitus and acute coronary syndrome (32). The upcoming large-scale prospective FAVOR III China trial may further elucidate the prognostic implications of QFR-guided PCI (33).

\section{Limitation}

There are some limitations in the current study. First, the selection bias may exist due to the retrospective nature of this single-center study. Second, acoustic shadow of calcification may prevent the deep attenuation from detecting by IVUS. Third, although the prognostic value of echo-attenuation has been demonstrated in the posthoc analysis of previous IVUS trials, prospective outcome data are still lacking (5). Additionally, QFR should also be validated against other intravascular imaging modalities including NIRS and OCT. Fourth, the invasive physiological references, as well as the accuracy and feasibility of QFR computation were limited because of the retrospective nature. Finally, despite being one of the most well investigated virtual functional index, the commercially availability of QFR machine remains limited worldwide, which may limit the generalizability of the findings of the current study (34).

\section{Conclusions}

In intermediate coronary lesions, presence of IVUS-derived echo-attenuation is associated with increased risk of QFRdefined functional significance. Further studies are warranted to determine if QFR assessment could be used to detect vulnerable plaque that could potentially benefit from PCI.

\section{Acknowledgments}

The authors appreciate Dr. Weifeng Shen for assisting with the preparation of the manuscript.

Funding: The research received grants from Leader Training Plan of Shanghai Pudong District (PWRd2018-06), Shanghai Key clinical specialty Project (shslczdzk06202), Key Disciplines Group Construction Project of Pudong Health Bureau of Shanghai (PWZxq2017-05), Toplevel Clinical Discipline Project of Shanghai Pudong District (PWYgf2018-02) and Jiangxi Municipal Health Commission (202120110).

\section{Footnote}

Reporting Checklist: The authors have completed the STARD Checklist. Available at https://dx.doi.org/10.21037/cdt-21402

Data Sharing Statement: Available at https://dx.doi. org/10.21037/cdt-21-402

Peer Review File: Available at https://dx.doi.org/10.21037/ 
cdt-21-402

Conflicts of Interest: All authors have completed the ICMJE uniform disclosure form (available at https://dx.doi. org/10.21037/cdt-21-402). The authors have no conflicts of interest to declare.

Ethical Statement: The authors are accountable for all aspects of the work in ensuring that questions related to the accuracy or integrity of any part of the work are appropriately investigated and resolved. The study protocol was applied in accordance with the Declaration of Helsinki (as revised in 2013) and was approved by the institutional review board of Shanghai East Hospital (research project number: 2020/No.007). Individual consent for this retrospective analysis was waived.

Open Access Statement: This is an Open Access article distributed in accordance with the Creative Commons Attribution-NonCommercial-NoDerivs 4.0 International License (CC BY-NC-ND 4.0), which permits the noncommercial replication and distribution of the article with the strict proviso that no changes or edits are made and the original work is properly cited (including links to both the formal publication through the relevant DOI and the license). See: https://creativecommons.org/licenses/by-nc-nd/4.0/.

\section{References}

1. Ciccarelli G, Barbato E, Toth GG, et al. Angiography Versus Hemodynamics to Predict the Natural History of Coronary Stenoses: Fractional Flow Reserve Versus Angiography in Multivessel Evaluation 2 Substudy. Circulation 2018;137:1475-85.

2. Park SJ, Kang SJ, Ahn JM, et al. Visual-functional mismatch between coronary angiography and fractional flow reserve. JACC Cardiovasc Interv 2012;5:1029-36.

3. $\mathrm{Pu}$ J, Mintz GS, Biro S, et al. Insights into echo-attenuated plaques, echolucent plaques, and plaques with spotty calcification: novel findings from comparisons among intravascular ultrasound, near-infrared spectroscopy, and pathological histology in 2,294 human coronary artery segments. J Am Coll Cardiol 2014;63:2220-33.

4. Tamaru H, Fujii K, Fukunaga M, et al. Impact of spotty calcification on long-term prediction of future revascularization: a prospective three-vessel intravascular ultrasound study. Heart Vessels 2016;31:881-9.

5. Shishikura D, Kataoka Y, Di Giovanni G, et al.
Progression of ultrasound plaque attenuation and low echogenicity associates with major adverse cardiovascular events. Eur Heart J 2020;41:2965-73.

6. Ahmadi A, Stone GW, Leipsic J, et al. Prognostic Determinants of Coronary Atherosclerosis in Stable Ischemic Heart Disease: Anatomy, Physiology, or Morphology? Circ Res 2016;119:317-29.

7. Xu B, Tu S, Qiao S, et al. Diagnostic Accuracy of Angiography-Based Quantitative Flow Ratio Measurements for Online Assessment of Coronary Stenosis. J Am Coll Cardiol 2017;70:3077-87.

8. Westra J, Tu S, Campo G, et al. Diagnostic performance of quantitative flow ratio in prospectively enrolled patients: An individual patient-data meta-analysis. Catheter Cardiovasc Interv 2019;94:693-701.

9. Tu S, Westra J, Adjedj J, et al. Fractional flow reserve in clinical practice: from wire-based invasive measurement to image-based computation. Eur Heart J 2020;41:3271-9.

10. Bär S, Kavaliauskaite R, Ueki Y, et al. Quantitative Flow Ratio to Predict Nontarget Vessel-Related Events at 5 Years in Patients With ST-Segment-Elevation Myocardial Infarction Undergoing Angiography-Guided Revascularization. J Am Heart Assoc 2021;10:e019052.

11. Zhang R, Song C, Guan C, et al. Prognostic Value of Quantitative Flow Ratio Based Functional SYNTAX Score in Patients With Left Main or Multivessel Coronary Artery Disease. Circ Cardiovasc Interv 2020;13:e009155.

12. Mintz GS, Nissen SE, Anderson WD, et al. American College of Cardiology Clinical Expert Consensus Document on Standards for Acquisition, Measurement and Reporting of Intravascular Ultrasound Studies (IVUS). A report of the American College of Cardiology Task Force on Clinical Expert Consensus Documents. J Am Coll Cardiol 2001;37:1478-92.

13. Inaba S, Mintz GS, Farhat NZ, et al. Impact of positive and negative lesion site remodeling on clinical outcomes: insights from PROSPECT. JACC Cardiovasc Imaging 2014;7:70-8.

14. Stone GW, Maehara A, Lansky AJ, et al. A prospective natural-history study of coronary atherosclerosis. N Engl J Med 2011;364:226-35.

15. DeLong ER, DeLong DM, Clarke-Pearson DL. Comparing the areas under two or more correlated receiver operating characteristic curves: a nonparametric approach. Biometrics 1988;44:837-45.

16. Ding D, Yang J, Westra J, et al. Accuracy of 3-dimensional and 2-dimensional quantitative coronary angiography for predicting physiological significance of coronary 
stenosis: a FAVOR II substudy. Cardiovasc Diagn Ther 2019;9:481-91.

17. Geng L, Yuan Y, Du P, et al. Association of quantitative flow ratio-derived microcirculatory indices with anatomical-functional discordance in intermediate coronary lesions. Int J Cardiovasc Imaging 2021;37:2803-13.

18. Waksman R, Legutko J, Singh J, et al. FIRST: Fractional Flow Reserve and Intravascular Ultrasound Relationship Study. J Am Coll Cardiol 2013;61:917-23.

19. Brown AJ, Giblett JP, Bennett MR, et al. Anatomical plaque and vessel characteristics are associated with hemodynamic indices including fractional flow reserve and coronary flow reserve: A prospective exploratory intravascular ultrasound analysis. Int J Cardiol 2017;248:92-6.

20. Obaid DR, Calvert PA, McNab D, et al. Identification of coronary plaque sub-types using virtual histology intravascular ultrasound is affected by inter-observer variability and differences in plaque definitions. Circ Cardiovasc Imaging 2012;5:86-93.

21. Driessen RS, Stuijfzand WJ, Raijmakers PG, et al. Effect of Plaque Burden and Morphology on Myocardial Blood Flow and Fractional Flow Reserve. J Am Coll Cardiol 2018;71:499-509.

22. Driessen RS, de Waard GA, Stuijfzand WJ, et al. Adverse Plaque Characteristics Relate More Strongly With Hyperemic Fractional Flow Reserve and Instantaneous Wave-Free Ratio Than With Resting Instantaneous WaveFree Ratio. JACC Cardiovasc Imaging 2020;13:746-56.

23. Kimura S, Sugiyama T, Hishikari K, et al. The clinical significance of echo-attenuated plaque in stable angina pectoris compared with acute coronary syndromes: A combined intravascular ultrasound and optical coherence tomography study. Int J Cardiol 2018;270:1-6.

24. Usui E, Yonetsu T, Murai T, et al. Prevalence of Thin-Cap Fibroatheroma in Relation to the Severity of Anatomical and Physiological Stenosis. Circ J 2017;81:1816-23.

25. Kanno Y, Sugiyama T, Hoshino M, et al. Optical coherence tomography-defined plaque vulnerability in relation to functional stenosis severity stratified by fractional flow reserve and quantitative flow ratio. Catheter Cardiovasc Interv 2020;96:E238-47.

26. Wu X, von Birgelen C, Li Z, et al. Assessment of superficial coronary vessel wall deformation and stress: validation of in silico models and human coronary arteries in vivo. Int J Cardiovasc Imaging 2018;34:849-61.

27. Wu X, von Birgelen C, Zhang S, et al. Simultaneous evaluation of plaque stability and ischemic potential of coronary lesions in a fluid-structure interaction analysis. Int J Cardiovasc Imaging 2019;35:1563-72.

28. Murai K, Kataoka Y, Nakaoku Y, et al. The association between the extent of lipidic burden and delta-fractional flow reserve: analysis from coronary physiological and near-infrared spectroscopic measures. Cardiovasc Diagn Ther 2021;11:362-72.

29. Pugliese L, Spiritigliozzi L, Di Tosto F, et al. Association of plaque calcification pattern and attenuation with instability features and coronary stenosis and calcification grade. Atherosclerosis 2020;311:150-7.

30. Shiono Y, Kubo T, Tanaka A, et al. Impact of attenuated plaque as detected by intravascular ultrasound on the occurrence of microvascular obstruction after percutaneous coronary intervention in patients with ST-segment elevation myocardial infarction. JACC Cardiovasc Interv 2013;6:847-53.

31. Kimura S, Sugiyama T, Hishikari K, et al. Association of Intravascular Ultrasound- and Optical Coherence Tomography-Assessed Coronary Plaque Morphology With Periprocedural Myocardial Injury in Patients With Stable Angina Pectoris. Circ J 2015;79:1944-53.

32. Kedhi E, Berta B, Roleder T, et al. Thin-cap fibroatheroma predicts clinical events in diabetic patients with normal fractional flow reserve: the COMBINE OCT-FFR trial. Eur Heart J 2021;42:4671-9.

33. Song L, Tu S, Sun Z, et al. Quantitative flow ratioguided strategy versus angiography-guided strategy for percutaneous coronary intervention: Rationale and design of the FAVOR III China trial. Am Heart J 2020;223:72-80.

34. Cortés C, Carrasco-Moraleja M, Aparisi A, et al. Quantitative flow ratio-Meta-analysis and systematic review. Catheter Cardiovasc Interv 2021;97:807-14.

Cite this article as: Geng L, Yuan Y, Du P, Gao L, Wang Y, Li J, Guo W, Huang Y, Zhang Q. The association between intravascular ultrasound-derived echo-attenuation and quantitative flow ratio in intermediate coronary lesions. Cardiovasc Diagn Ther 2021;11(6):1206-1216. doi: 10.21037/ cdt-21-402 